\title{
Effect of combined chronic predictable and unpredictable stress on depression-like symptoms in mice
}

\author{
Yajun Qiao ${ }^{1,2,3 \#}$, Jiubo Zhao ${ }^{1,4 \#}$, Cen $\mathrm{Li}^{2,5}$, Ming Zhang ${ }^{2,5}$, Lixin Wei ${ }^{2,5}$, Xiaoyuan Zhang ${ }^{1,4}$, Olga Kurskaya ${ }^{6}$, \\ Hongtao $\mathrm{Bi}^{2}$, Tingting Gao ${ }^{1,4}$ \\ ${ }^{1}$ Department of Psychology, School of Public Health, Southern Medical University, Guangzhou, China; ${ }^{2}$ Qinghai Provincial Key Laboratory of \\ Tibetan Medicine Pharmacology and Safety Evaluation, Northwest Institute of Plateau Biology, Chinese Academy of Science, Xining, China; \\ ${ }^{3}$ University of Chinese Academy of Sciences, Beijing, China; ${ }^{4}$ Department of Psychiatry, Zhujiang Hospital, Southern Medical University, \\ Guangzhou, China; ${ }^{5}$ CAS Key Laboratory of Tibetan Medicine Research, Northwest Institute of Plateau Biology, Chinese Academy of Sciences, \\ Xining, China; ${ }^{6}$ Department of Experimental Modeling and Pathogenesis of Infectious Diseases, Federal Research Center of Fundamental and \\ Translational Medicine, Novosibirsk, Russia \\ Contributions: (I) Conception and design: H Bi, T Gao; (II) Administrative support: L Wei, X Zhang, H Bi, T Gao; (III) Provision of study materials \\ or patients: Y Qiao, J Zhao, C Li, M Zhang; (IV) Collection and assembly of data: Y Qiao, L Wei, H Bi; (V) Data analysis and interpretation: X \\ Zhang, O Kurskaya; (VI) Manuscript writing: All authors; (VII) Final approval of manuscript: All authors. \\ \#These authors contributed equally to this work. \\ Correspondence to: Xiaoyuan Zhang. Department of Psychiatry, Zhujiang Hospital, Southern Medical University, 253 Middle Gongye Ave, \\ Guangzhou 510282, China. Email: zhxy@fimmu.com; Hongtao Bi. Qinghai Provincial Key Laboratory of Tibetan Medicine Pharmacology and \\ Safety Evaluation, Northwest Institute of Plateau Biology, CAS, 23 Xinning Road, Xining 810008, China. Email: bihongtao@hotmail.com; Tingting \\ Gao. Department of Psychology, School of Public Health, Southern Medical University, 1023 South Shatai Road, Guangzhou 510515, China. \\ Email: gaott646@hotmail.com.
}

Background: Mental stress mainly induces depression, and predictable stress, as well as a constant bombardment of chronic unpredictable micro-stressors, always coexist in daily life. However, the combined effect of predictable and unpredictable stress on depression is still not fully understood.

Methods: The chronic restraint stress (CRS) is to restrain the mice for $6 \mathrm{~h}$ per day for 3 weeks, and the chronic unpredictable mild stress (CUMS) is to stimulate the mice with 7 different stressors for 3 weeks. We evaluated the combined effect of CRS and CUMS on depression-like symptoms using behavioral tests and investigated the action mechanism through analysis of neurotransmitters, brain-derived factors, inflammatory factors, antioxidants, and intestinal microorganisms.

Results: Our data suggested the combined stress of CRS and CUMS caused significant weight loss, food intake reduction, depression-like behaviors-including anhedonia, learned helplessness, and reduction in spontaneous activity — and even atrophy and severe structural damage to the hippocampus in mice. Our pathogenesis study showed that combined stress-induced the reduction of glucocorticoid receptor (GR) levels, loss of oligodendrocytes (NG2 and Olig2 cells), and inhibition of neuron proliferation in the CA1, CA3, and DG regions of the hippocampus, decreased the contents of monoamine neurotransmitters (5HT and NE) and BDNF in the cerebral cortex, caused hyperactivity of the HPA system, led to immune dysfunction, aggravated oxidative stress, and weakened the capacity of antioxidants in mice. Compared with single stress, combined stress gave rise to a more significant diversity change of the gut microbiota.

Conclusions: Combined stress caused significant depression-like behaviors, atrophy, and severe structural damage to the hippocampus in mice via monoamine neurotransmitter, BDNF, HPA axis, neurogenesis, and neurodegenerative, immune, oxidative stress and gut-brain axis action pathways.

Keywords: Depression; predictable stress; chronic unpredictability mild stress; combined stress; chronic bondage stress 
Submitted Jun 09, 2020. Accepted for publication Jul 29, 2020.

doi: $10.21037 /$ atm-20-5168

View this article at: http://dx.doi.org/10.21037/atm-20-5168

\section{Introduction}

Depression is a common mood disorder whose typical symptoms include low mood, slow thinking, loss of interest, cognitive impairment, eating and sleeping disorders, and other physical symptoms (1). Depression has become one of the most common diseases affecting human physical and mental health in recent years (2). However, we do not yet fully understand the causes and pathogenesis of depression. According to the world health organization (WHO), around 350 million people suffer from depression to some degree and it is expected to become the world's leading disease burden by 2030 (3). Studies on sequential treatment of depression show that residual symptoms, such as depressed mood, impaired function and negative concept, still exist in patients with complete remission after treatment (4), and patients with residual symptoms have a higher recurrence rate and a shorter recurrence interval (5).

According to the etiological studies, mental stress mainly induces depression, repeated physical illness, and genetic factors, with more than half of cases caused by the first factor (6). With these causes, researchers have developed models of depression with factors including stress, surgery, postpartum depression, and genetics, among which the chronic unpredictable mild stress (CUMS) model and chronic restraint stress (CRS) model are two of the most commonly used stress-induced depression models $(7,8)$. CUMS model is constructed using chronic unpredictable low levels of stressful stimulation over a prolonged period (9), while CRS deprives animals of their freedom predictably (10). Both models simulate the environment of depression like the human stress disorder depression in real life and can cause depression-like symptoms to different degrees, and the inhibition of exploratory behavior is apparent, which can lead to typical behavioral despair $(11,12)$. However, predictable stress and a constant bombardment of chronic unpredictable micro-stressors always coexist in daily life, and the combined effect of predictable and unpredictable stress on psychiatric states is still not fully understood. Therefore, to investigate the combined action of predictable and unpredictable stress on psychiatric states, we evaluated the effect of the combined stress of CUMS and CRS on depression-like symptoms using behavioral tests and investigated the action mechanism through analysis of the neurotransmitters, brain-derived factors, inflammatory factors, antioxidants, and intestinal microorganisms in the present study. Compared with the above published studies, the novel ideas of the present study are manly in three aspects: (I) the present study focuses on the combined action of CRS and unpredictable stress CUMS instead of their single action on depression-like symptoms in mice; (II) we evaluated the effect of the combined stress of CUMS and CRS on depression-like symptoms not only mainly focusing on the changes in behavioral tests; (III) to our best knowledge, the present study reports the effect of the combined stress of CRS and CUMS on the intestinal microbial diversity in mice for the first time. We present the following article in accordance with the ARRIVE reporting checklist (available at http://dx.doi.org/10.21037/atm-205168).

\section{Methods}

\section{Material}

The enzyme-linked immunosorbent assay (ELISA) kits of 5-hydroxytryptamine (5-HT), norepinephrine (NE), brain-derived neurotrophic factor (BDNE), corticosterone $(\mathrm{CORH})$, corticotropin-releasing hormone (CRH), adrenocorticotropic hormone (ACTH), interleukin-6 (IL-6), interleukin-10 (IL-10), and tumor necrosis factor- $\alpha(\mathrm{TNF} \alpha)$ were purchased from Jianglai Biotechnology Co., Ltd. (Shanghai, China). The assay kits of superoxide dismutase (SOD) and catalase (CAT) and malondialdehyde (MDA) were bought from Nanjing Jiancheng Bioengineering Institute (Nanjing, China). Anti-glucocorticoid receptor (GR) antibody (ab3578), anti-PCNA antibody (ab18197), anti-NeuN antibody (ab128886), anti-NG2 antibody (ab129051), and anti-Olig2 antibody (ab136253) were procured from Abcam, Inc. (Cambridge, Cambridge shire, UK). All the other chemicals were of analytical grade manufactured in China.

\section{Animals and treatment}

Male KM mice at the age of 8 weeks were obtained from the Gansu University of Chinese Medicine, China (animal production license number: SCXK [Gan] 2015-0005). The 
mice were housed in standard cages with wood shavings in a room with carefully controlled ambient temperature $\left(22 \pm 1{ }^{\circ} \mathrm{C}\right)$ and artificial lighting from 7:00 am to 7:00 pm and were fed standard laboratory chow and distilled water ad libitum. All the animal experiments were in compliance with the ARRIVE guidelines, carried out in strict accordance with the National Institutes of Health Guide for the Care and Use of Laboratory Animals (NIH Publication No. 8023, revised 1978), and approved by the committee of the Northwest Plateau Institute of Biology, CAS, for animal experiments (allowance number: NWIPB20171106-01).

After one-week acclimatization, the mice were randomly divided into 4 groups ( $n=10$ ): (I) normal, (II) CRS, (III) CUMS, and (IV) combined stress (CRS + CUMS). Mice in the CRS group were placed in the tubes from 8:30 to 14:30 for 3 weeks (13). The CUMS mice were exposed to 7 different stressors for 3 weeks: 5 minutes of heat stress at $45{ }^{\circ} \mathrm{C}, 2$ minutes of cold stress at $10^{\circ} \mathrm{C}, 2$ minutes of reciprocating sway, 24 hours of $45^{\circ}$ tilted cages and humid environment, 24 hours of food deprivation, 24 hours of water deprivation, and 24 hours of day and night reversal. Each stimulation was randomly arranged and performed three times per day (14). Mice in the combined stress group were exposed to daily confinement for 6 hours $(8: 30-14: 30)$ combined with unforeseeable mild stress from 7 different stressors for 3 weeks. The body weights of the mice were measured weekly during the stress stimulation process.

\section{Behavioral tests}

The behavioral tests, including the sucrose preference test (SPT), open field test (OFT), tail suspension test (TST) and forced swimming test (FST) were conducted before the CRS, CUMS and CRS + CUMS procedure began (0 days) and at the end of the 3 -week stress period (21 days). SPT was carried out following the procedures described by Dang et al. (15), and the sucrose preference ratio (SPR) was calculated as follows: SPR $(\%)=$ sucrose intake/total fluid intake (including sucrose and water intake). OFT was performed following the procedures described by Choleris et al. (16). TST was performed following the procedures described by Steru et al. (17). FST was conducted following the procedures described by Porsolt et al. (18).

\section{Determination of biomarkers related to depression, immune function, and oxidative stress}

After behavioral tests, mouse blood was collected using the posterior orbital venous plexus, and then the mouse was at once sacrificed by cervical dislocation. Mouse blood was placed into a coagulation tube, and the serum was obtained by centrifugation at 4,000 RPM for 10 minutes at $4{ }^{\circ} \mathrm{C}$. The cortex and hypothalamus were respectively detached from the brain, mixed with the phosphatebuffered solution ( $\mathrm{pH}$ 7.2), and homogenized on ice using a tissue homogenizer (frequency: $60 \mathrm{~Hz}$, rotation rate: 1,800 times/minutes, 2 minutes). After centrifugation at 5,000 RPM for 10 minutes, the supernatant was separated from the cortex or hypothalamus homogenate. 5-HT and NE contents of the cortex supernatant, BDNF content of the hypothalamus supernatant, serum Cort and ACTH levels, and hypothalamus CRH content were determined by using ELISA kits following their operating instructions. The contents of TNF- $\alpha$, IL-6, IL-10, MDA, CAT, and SOD in serum were determined by ELISA kits following their operating instructions.

\section{Immunofluorescence histochemistry assay}

Whole brains were collected from the control and stress stimulation groups after the stress simulations were done. Briefly, the brains were fixed in $4 \%$ paraformaldehyde solution, paraffin-embedded, and serially sectioned at a thickness of $5 \mu \mathrm{m}$. Then, the paraffin-embedded sections were deparaffinized, and non-specific binding was blocked by incubation with $3 \%$ BSA for 30 minutes. Subsequently, brain sections were incubated with primary antibodies against PCNA (1:500), GR (1:500), NG2 (1:500), NeuN (1:200), and Olig-2 (1:250) overnight at $4{ }^{\circ} \mathrm{C}$. After incubation and washing, FITC-conjugated and Cy3conjugated secondary antibodies were added. Following incubation with the antibodies $\left(4{ }^{\circ} \mathrm{C}, 24\right.$ hours for primary antibodies and room temperature, 45 minutes for secondary antibodies), the sections were embedded in Fluoroshield mounting medium with DAPI (ab104139, Abcam). Images were acquired using fluorescent microscopy (IX73, Olympus, Japan).

\section{Intestinal microbial diversity analysis}

After the stress stimulations, fresh rectal contents of mice were collected, frozen quickly in liquid nitrogen, and placed in a refrigerator at $-80{ }^{\circ} \mathrm{C}$ for DNA extraction. Microbial DNA was extracted using the EZNA Stool DNA Kit (Omega Bio-tek, Norcross, GA, USA) according to the manufacturer's protocols and then stored at $-80^{\circ} \mathrm{C}$. 
Primers 338F (5'-CTCCTACGGGAGGCAGCAG-3') and 806R (5'-GGACTACHVGGGTWTCTAAT-3') were used to amplify the V3-V4 region of the bacterial $16 \mathrm{~S}$ rDNA gene. The amplification procedure of PCR reactions was $95{ }^{\circ} \mathrm{C}$ for 3 minutes, followed by 27 cycles at $95^{\circ} \mathrm{C}$ for $30 \mathrm{~s}, 55^{\circ} \mathrm{C}$ for $30 \mathrm{~s}$, and $72{ }^{\circ} \mathrm{C}$ for $45 \mathrm{~s}$ and a final extension at $72{ }^{\circ} \mathrm{C}$ for 10 minutes; about $420 \mathrm{bp}$ amplified fragments were obtained. The PCR reactions were performed in a $20-\mu \mathrm{L}$ mixture containing $4 \mu \mathrm{L}$ of $5 \times$ FastPfu Buffer, $2 \mu \mathrm{L}$ of $2.5 \mathrm{mM}$ dNTPs, $0.8 \mu \mathrm{L}$ of $5 \mu \mathrm{M}$ primers, $0.4 \mu \mathrm{L}$ of FastPfu Polymerase, and $10 \mathrm{ng}$ of template DNA using a PCR system (ABI GeneAmp 9700, Applied Biosystems, Inc., MA, USA). Paired-end data of $2 \times 300 \mathrm{bp}$ were obtained by sequencing using the MiSeq platform. Splicing could obtain long sequences, and 16S analysis was performed. The raw reads were deposited into the NCBI Sequence Read Archive (SRA) database.

The original sequence was quality-filtered using Trimmomatic software (version 0.39) and spliced by FLASH software with the following criteria: (I) setting a $50 \mathrm{bp}$ sliding window. The $300 \mathrm{bp}$ reads were truncated at any site receiving an average quality score $<20$ over this window, discarding the truncated reads that were shorter than 50 bp; (II) bar codes matching exactly-2-nucleotide mismatch in primer matching was allowed, and reads containing ambiguous characters were removed; (III) two end sequences were spliced according to base overlap, the overlap was longer than $10 \mathrm{bp}$, and unspliced sequences were removed. The reads that could not be assembled were discarded. Operational taxonomic units (OTUs) were clustered with $97 \%$ similarity cutoff using UPARSE (version $7.1 \mathrm{http}: / /$ drive 5.com/uparse/), and chimeric sequences were identified and removed using UCHIME. The taxonomy of each $16 \mathrm{~S}$ rRNA gene sequence was analyzed by RDP Classifier (http://rdp.cme.msu.edu/) against the Silva (SSU123)16S rRNA database using a confidence threshold of $70 \%(19)$.

\section{Statistical analysis}

All the data were expressed as the mean \pm standard deviation (SD) of six replicates and subjected to analysis of variance for a completely randomized experimental design. Multiple $t$-tests were conducted to identify differences among means (without assuming consistent SD) using SPSS 22.0 software. $\mathrm{P}<0.05$ was considered statistically significant.

\section{Results}

\section{Stress stimulations induced body weight loss}

As shown in Figure 1A, all the mice in the stress stimulation groups lost weight. Compared to the control group, the bodyweight losses of mice were $25.85 \%, 26.28 \%$ and $17.05 \%$ in the CRS group, $22.23 \%, 22.70 \%$ and $19.07 \%$ in the CUMS group, $17.05 \%, 17.20 \%$ and $19.52 \%$ in the CS group after stress stimulation for 1, 2, and 3 weeks, respectively. In the three stress stimulation groups, the bodyweight of mice in the CS group was the lowest $(\mathrm{P}<0.001)$ on day 22.

\section{Stress stimulations decreased the sucrose preference ratio of mice}

Anhedonia, as a core symptom of depression, is reflected by SPR and food consumption. As shown in Figure 1B,C, the SPR and 2-hours food consumption of mice in the stress stimulation groups dramatically declined after 3-week stress stimulation by $23.10 \%$ and $38.07 \%$ in CRS group, $25.04 \%$ and $21.83 \%$ in the CUMS group, and $28.96 \%$ and $30.46 \%$ in CS group compared to control group. Moreover, during the 3-week stress stimulation, SPRs of mice in three tested groups were all time-dependent and lowest on day 14. SPR of mice in the CS group was lowest in all tested groups during the test time, suggesting that combined stress has the most significant negative effect on anhedonia in mice.

\section{Stress stimulations induced increased immobility time in tail suspension test and forced swimming test}

Tail suspension test and forced swimming, two of the main methods to evaluate the despair behavior of mice, were used to investigate the effect of stress stimulations on the despair of mice. As shown in Figure 1D, 3-week stress stimulations significantly decreased the immobility time of mice in the tail suspension test and forced swimming test $(\mathrm{P}<0.05)$. Compared with the control group, the CS group showed a $91.93 \%$ and $348.21 \%$ increase in immobility time in the tail suspension test and forced swimming test. Moreover, the 

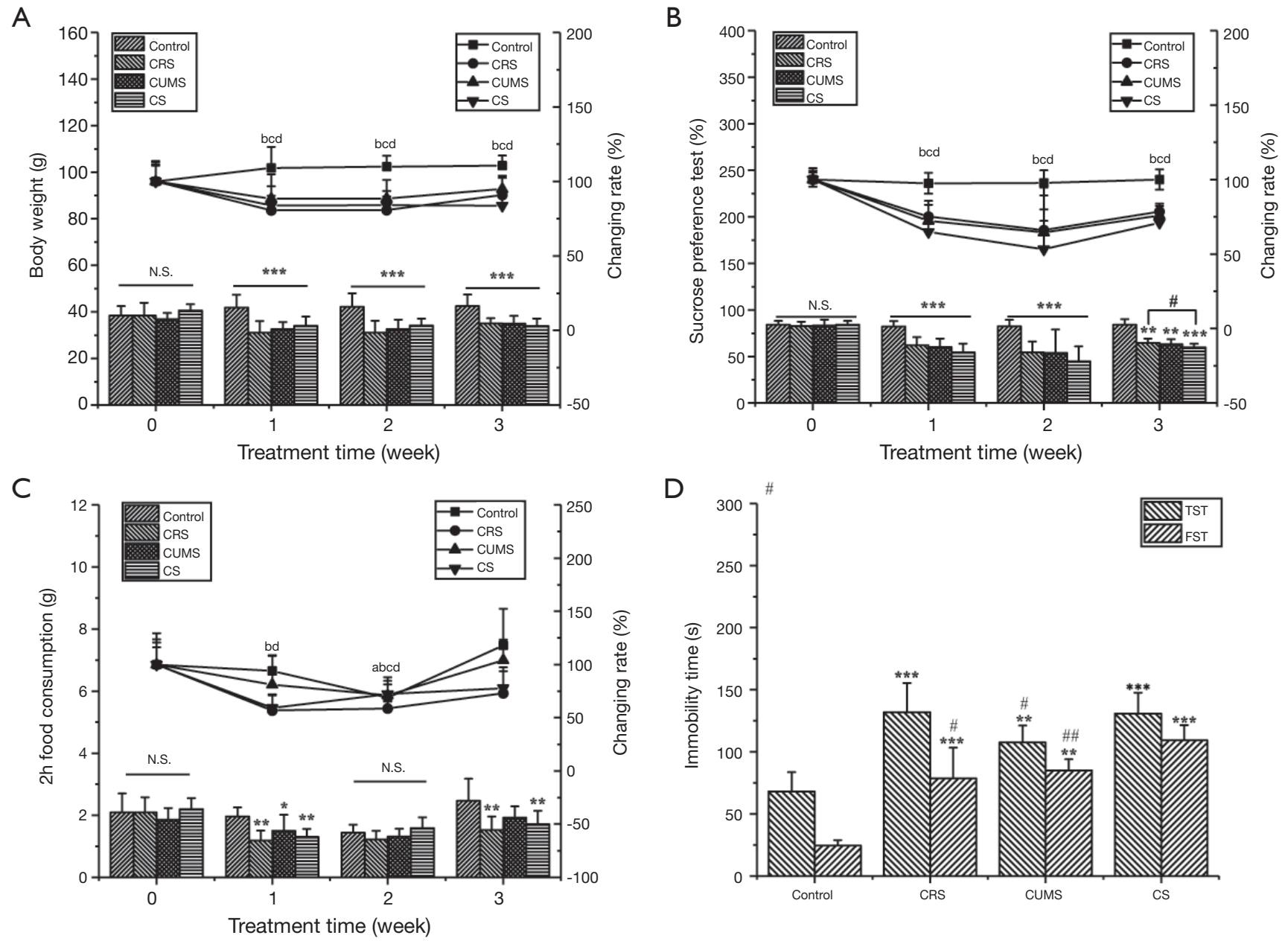

Figure 1 The effect of stress stimulations on (A) body weight (B) sucrose preference ratio, (C) 2-hours food consumption, and (D) immobility time in the tail suspension test (TST) and forced swimming test (FST) of mice. Data are shown as mean \pm SD $(\mathrm{n}=8)$. ${ }^{*}, \mathrm{P}<0.05$; ${ }^{* *}, \mathrm{P}<0.01$ and ${ }^{* * *}, \mathrm{P}<0.001$ denote significant differences from the control group simultaneously;, $\mathrm{P}<0.05$ and ${ }^{\# \#}, \mathrm{P}<0.01$ denote a significant difference from the CS group simultaneously. The lowercase letters denote significant difference $(\mathrm{P}<0.05)$ from the corresponding group on Day 0 of each group. NS, not significant.

immobility time of the CS group in TST was significantly longer than either CRS or CUMS group $(\mathrm{P}<0.05)$.

\section{Stress stimulations induced increased rest time and decreased center residence time and movement distance in open field test}

The activity of mice was evaluated using the OFT after 1-3-week stress treatment. As shown in Figure 2, compared to mice in the control group on Day 22 and the relative stress treatment group on Day 0, the rest time (RT) of mice in the stress treatment groups was significantly prolonged $(\mathrm{P}<0.05)$, and their movement time (MT), center residence time (CRT), and movement distance (CMD) were significantly shortened $(\mathrm{P}<0.05)$, indicating that the activity of mice was significantly reduced by 3 -week stress stimulations. During 1-3-week stress treatment, RTs of three tested groups were all increased with treatment time. The CRT of the CS group showed no significant change after 1 week, which was like the CUMS group but different from the CRS group (the CRT of the CRS group decreased with treatment time). The CMD of the CS group decreased with treatment time, which was different from the CRS and CUMS groups (the CMD of CRS group decreased first, then increased, and 
A

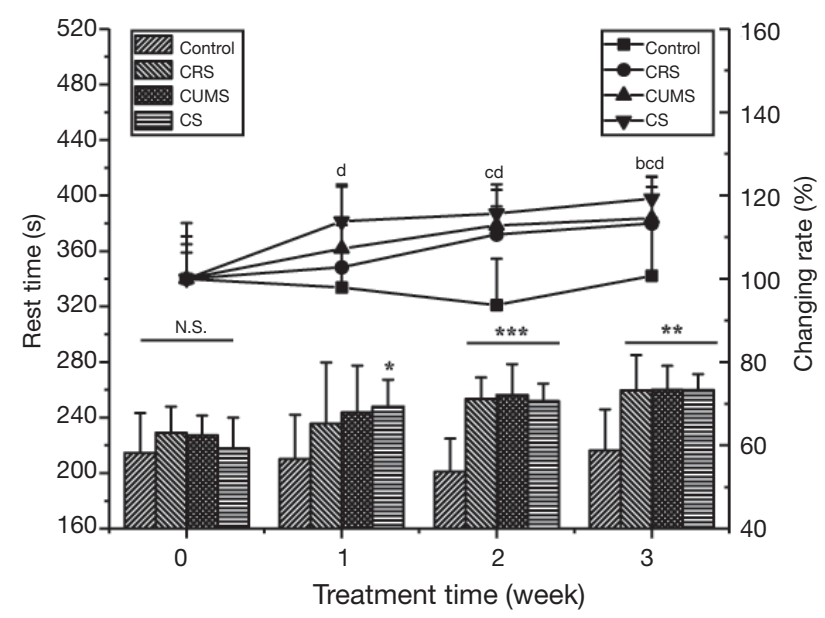

C

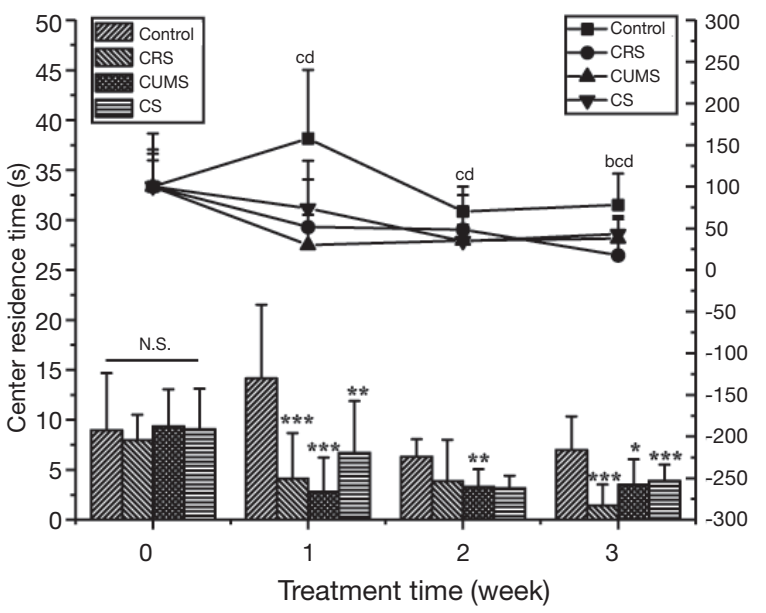

B

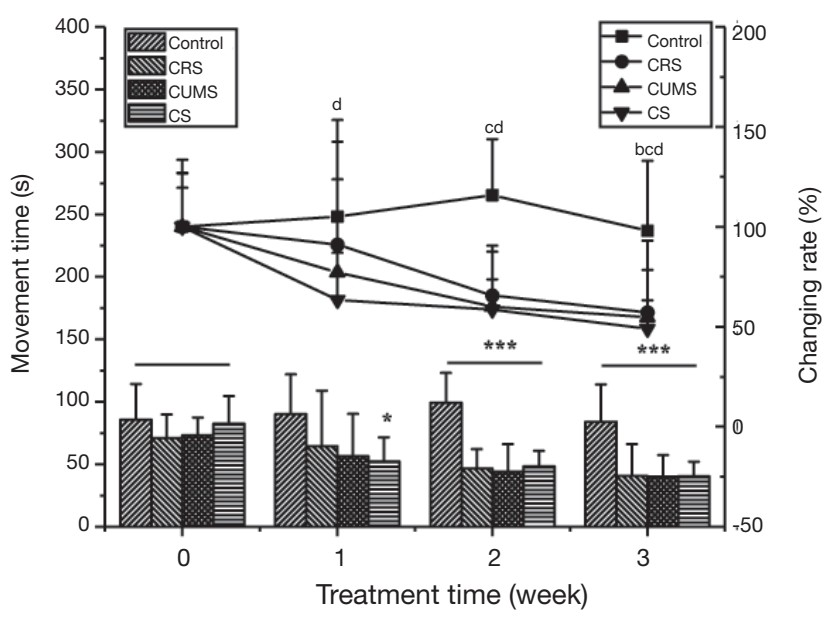

D

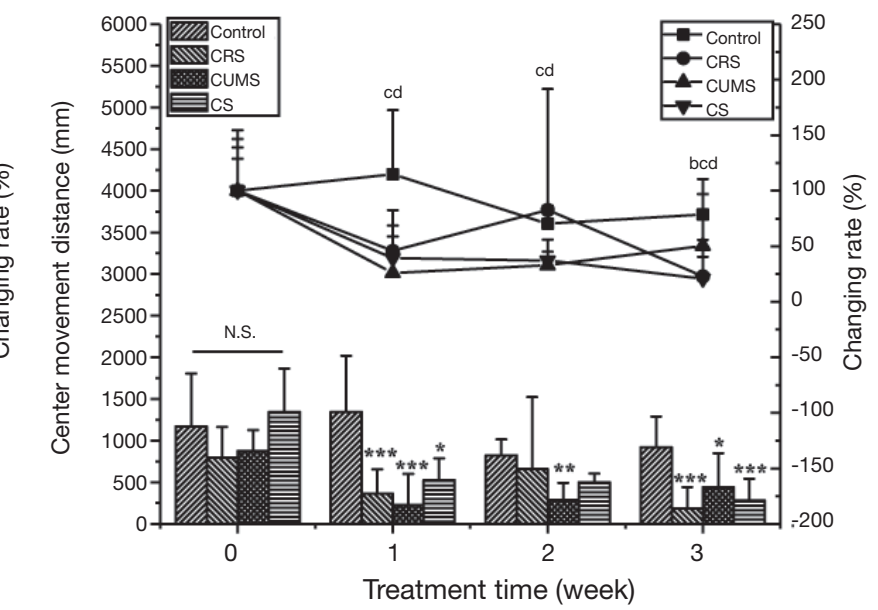




\section{$E$}

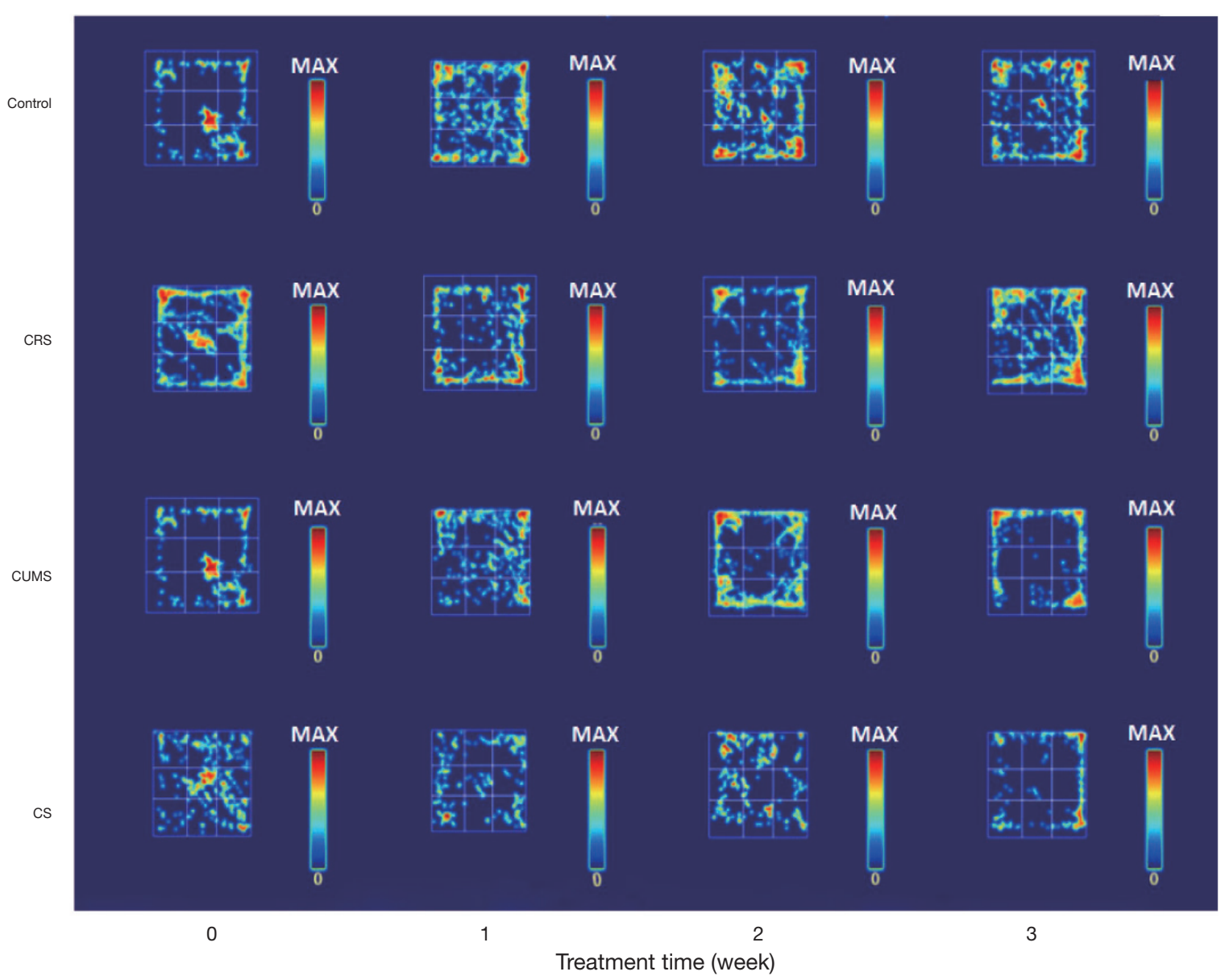

Figure 2 The effect of stress stimulations on depression-like behaviors of mice in the open-field test. (A) Rest time, (B) movement time, (C) central residence time, (D) center movement distance, (E) motion trajectory map of each group of mice in the OFT. Data are shown as mean $\pm \mathrm{SD}(\mathrm{n}=8) .{ }^{*}, \mathrm{P}<0.05,{ }^{* *}, \mathrm{P}<0.01$ and ${ }^{* * *}, \mathrm{P}<0.001$ denote significant difference from the control group simultaneously; the lowercase letters denote significant difference $(\mathrm{P}<0.05)$ from the corresponding group on day 0 of each group. NS, not significant.

finally decreased, while the CMD of CUMS decreased first and then increased slightly after 1 week).

\section{Stress stimulations induced a decrease of monoamine neurotransmitter and BDNF content and an increase of neuroendocrine bormone content in the HPA axis}

Levels of monoamine neurotransmitters 5 -HT and NE in the cortex, BDNF in the hippocampus, and three core neuroendocrine hormones (CRH, ACTH, and CORT) in HPA axis were determined, and the results are shown in Figure 3A,B,C. After 3-week stress stimulation, levels of $5-\mathrm{HT}$ and NE in cortex and BDNF in the hippocampus of stress treated mice were significantly lower than normal control mice $(\mathrm{P}<0.05)$. In the CS group, the contents of 5-HT, NE, and BDNF were $24.09 \%, 25.37 \%$, and $17.04 \%$ 


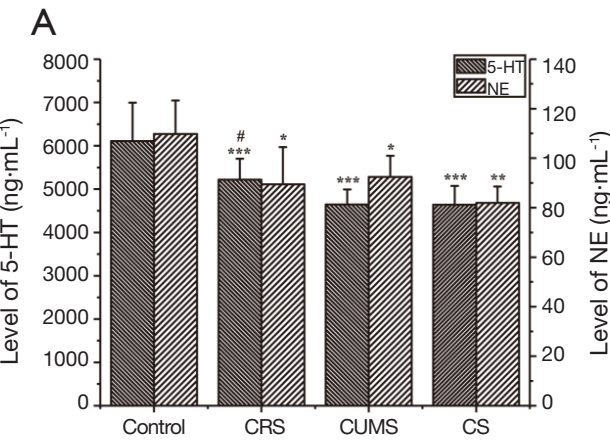

B

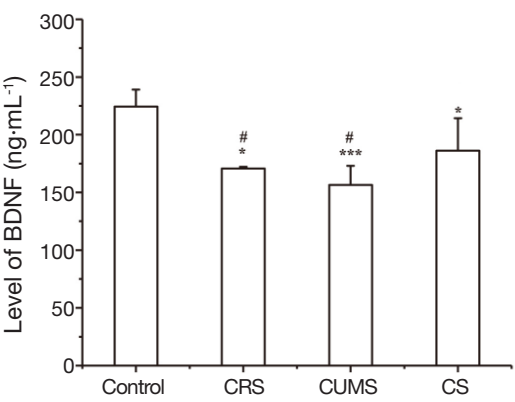

$\mathrm{E}$

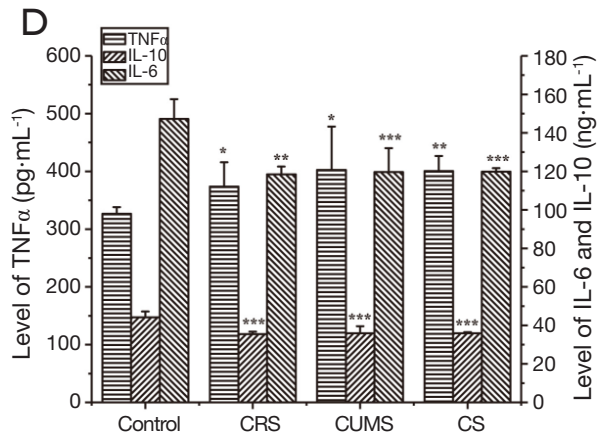

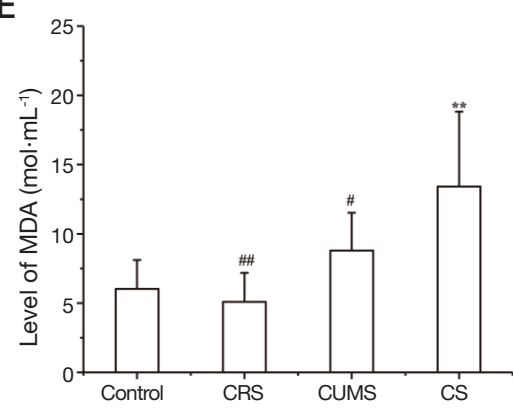

C

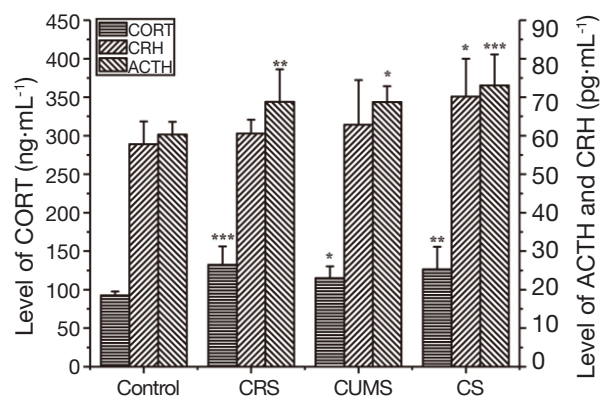

$\mathrm{F}$

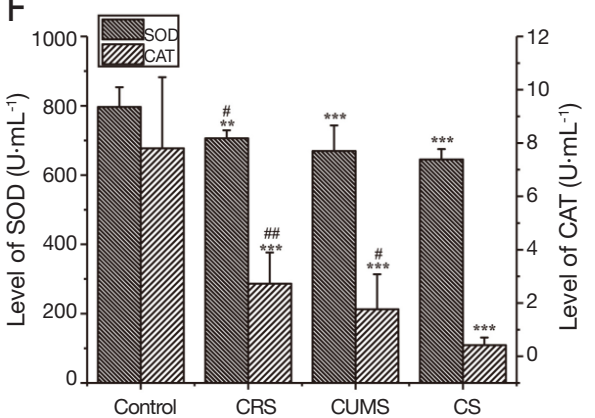

Figure 3 The effect of stress stimulations on biomarkers related to depression, immune factors, and oxidative stress of mice. (A) Monoamine neurotransmitter, (B) brain-derived neurotrophic factor (BDNF), (C) HPA axis correlation biomarker, (D) immune factors, (E) MDA, (F) antioxidases content of each group of mice. Data are shown as mean $\pm \mathrm{SD}(\mathrm{n}=8)$. ${ }^{*}, \mathrm{P}<0.05,{ }^{* *}, \mathrm{P}<0.01$ and ${ }^{* * *}, \mathrm{P}<0.001$ denote significant difference from the control group; ${ }^{\#}, \mathrm{P}<0.05$ and ${ }^{\# \#}, \mathrm{P}<0.01$ denote a significant difference from the CS group.

those of the control group, respectively. However, the levels of core neuroendocrine hormones (CRH, ACTH, and Cort) in the HPA axis of stress-treated mice were significantly higher than those of normal control mice $(\mathrm{P}<0.05)$. Compared with the control group, the contents of CRH, ACTH, and CORT were increased by $21.44 \%, 21.23 \%$, and $36.48 \%$, respectively, in the CS group. Moreover, in three stress-induced groups, the BDNF level of CS group was the highest, showing that CS had the weakest effect on inhibition of BDNF release.

\section{Stress stimulations induced the increase of TNF- $\alpha$ content}

\section{and the decrease of IL-6 and IL-10 content in serum}

The effect of stress on the immune-inflammatory response in mice was investigated according to TNF- $\alpha$, IL-6, and IL10 content in serum. As shown in Figure 3D, after 3-week stress stimulation, the TNF- $\alpha$ content in tested groups was significantly higher, while the contents of IL-6 and IL10 were both lower than the control group $(\mathrm{P}<0.05)$. The contents of TNF- $\alpha$, IL-6, and IL-10 in the CS group were
$22.59 \%, 18.55 \%$. Further, $23.06 \%$ of those in the control group, respectively, with the IL-10 level being lowest in all the groups.

\section{Stress stimulations induced aggravation of oxidative stress}

To evaluate the oxidative stress level of mice induced by 3-week stress stimulations, MDA, SOD, and CAT contents in serum were determined, and the results are shown in Figure $3 E, F$. Compared with the control group, MDA content was significantly higher in the CUMS group and CS group, and SOD and CAT contents were remarkably lower in three stress stimulation groups $(\mathrm{P}<0.05)$. Moreover, among the three-stress stimulation groups, the MDA level was the highest $(\mathrm{P}<0.05)$, and SOD and CAT levels were the lowest in the CS group $(\mathrm{P}<0.05)$.

Combined stress-induced structural damage, a decrease of GR and oligodendrocytes, and reduction of neuron proliferation in key bippocampal subregions

The effects of stress treatment on morphological structure, 
GR level, oligodendrocyte loss, and neuron proliferation in key hippocampal subregions were assessed using immunofluorescence histochemical staining, and the results are shown in Figure 4. The coronal section of the mouse brain shows complete morphology, orderly arrangement, clear structure, and deep staining degree of cells in each subregion of the hippocampus of mice in the control group as well as the CUMS group (Figure 4A). However, in CRS and CS groups, the arrangement of vertebral cells was disordered, the number of vertebral cells was decreased, and atrophy was clear in the hippocampus, suggesting severe structural damage.

The images of immunofluorescence staining of GR in key hippocampal subregions are shown in Figure 4B, and the fluorescence density results are shown in Figure 4B,C. Compared with the control group, the distribution range of GR-positive cells was narrowed, the fluorescence intensity was weakened, and cell morphology was irregular and disorderly in CRS and CR groups. In contrast, in the CUMS group, GR-positive cells showed a more extensive distribution range and higher fluorescence intensity in the CA1, CA3, and DG regions of the hippocampus.

We then assessed whether stress treatment causes a loss of oligodendrocytes in the hippocampus by measuring the levels of NG2 (a marker for oligodendrocyte progenitor cells) and Olig2 (a pan-oligodendrocyte marker). Following stress treatment, there was a dramatic loss of $\mathrm{NG} 2+$ and Olig2 + cells in the CA1, CA3, and DG regions of the hippocampus in CS group mice (Figure $4 D$ ). The fluorescence densities of NG2 + and Olig2 + cells in the CS group were $31.55 \%$ and $31.98 \%, 29.89 \%$ and $29.75 \%$, and $39.75 \%$ and $27.81 \%$ lower in the CA1, CA3, and DG regions, respectively compared with the control group (Figure 4E,F). Although the fluorescence density of NG2+ and Olig2+ cells in the above three hippocampal subregions was higher in the CUMS group than in the control group, the difference was insignificant.

Neuron proliferation in key hippocampal subregions was evaluated using cell cycle marker proliferating cell nuclear antigen (PCNA) and neuronal nuclear antigen neuron marker (NeuN). As shown in Figure $4 G$, the least intense reaction for PCNA immunoreactivity in DG was observed in the CS group, with reactivity $63.38 \%$ lower than the control group $(\mathrm{P}<0.05)$ (Figure $4 H)$. However, PCNA immunoreactivity increased significantly in CA1 in the CUMS group and CA3 in CRS groups compared to the control group. Immature and mature neurons in CA1, CA3, and DG were assessed by NeuN immunoreactivity.
As shown in Figure 4G,I, there was a significant increase of NeuN immunoreactivity in the CUMS group and a significant decrease in CRS and CS groups in the above hippocampal subregions $(\mathrm{P}<0.05)$.

\section{Stress influences the gut microbiota}

Analysis of alpha diversity revealed no significant differences between normal mice and stress-treated mice. However, the analysis of the beta diversity calculated from the unweighted and weighted UniFrac distances and the Bray-Curtis dissimilarity revealed that the bacterial microbiota of stresstreated mice clustered apart from normal mice $[\mathrm{P}<0.05$, multi-response permutation procedure (MRPP) analysis; Figure 5 and Table 1], and the diversity of the gut microbiota also differed significantly among the three stress-treated groups $(A>0$ and $\mathrm{P}<0.05)$, indicating varying degrees of significant effects on the diversity of gut microbiota caused by the three stress stimulations. The phylumlevel composition of the gut microbiota was identified (Figure 6A), and the differences in gut microbiota abundance between the control group and stress-treated groups were analyzed by $t$-test (Figure $6 B, C$ ). The result showed that the relative abundance of Firmicutes and Verrucomicrobia was significantly different $(\mathrm{P}<0.05)$ in the CS group, and the relative abundance of Tenericutes was significantly different $(\mathrm{P}<0.05)$ in CUMS group compared to control group. However, there was no significant difference in gut microbiota at the phylum level between the CRS and control groups.

The order levels of gut microbiota were investigated (Figure 7A), and Clostridiales and Verrucomicrobiales were present at significantly higher and lower levels, respectively, in the CS group compared with the control group $(\mathrm{P}<0.05)$ (Figure $7 B)$. Compared with the control group, two orders (Mycoplasmatales and Lactobacillales) and one order (Mycoplasmatales) of bacteria were significantly lower $(\mathrm{P}<0.05)$ in CUMS and CRS groups, respectively (Figure $7 C, D)$.

The family levels of gut microbiota were assayed (Figure 8A). Compared with the control group, two families of gut microbiota (Ruminococcaceae and Marinifilaceae) were significantly higher $(\mathrm{P}<0.05$ or $\mathrm{P}<0.01)$ and one family (Bacteriaceae) was significantly lower $(\mathrm{P}<0.01)$ in relative abundance in CS group (Figure $8 B$ ). Additionally, one family (Muribaculaceae) and two families (Muribaculaceae and Lactobacillaceae) of gut microbiota were significantly lower $(\mathrm{P}<0.05$ and $\mathrm{P}<0.01)$ in relative abundance in CRS 

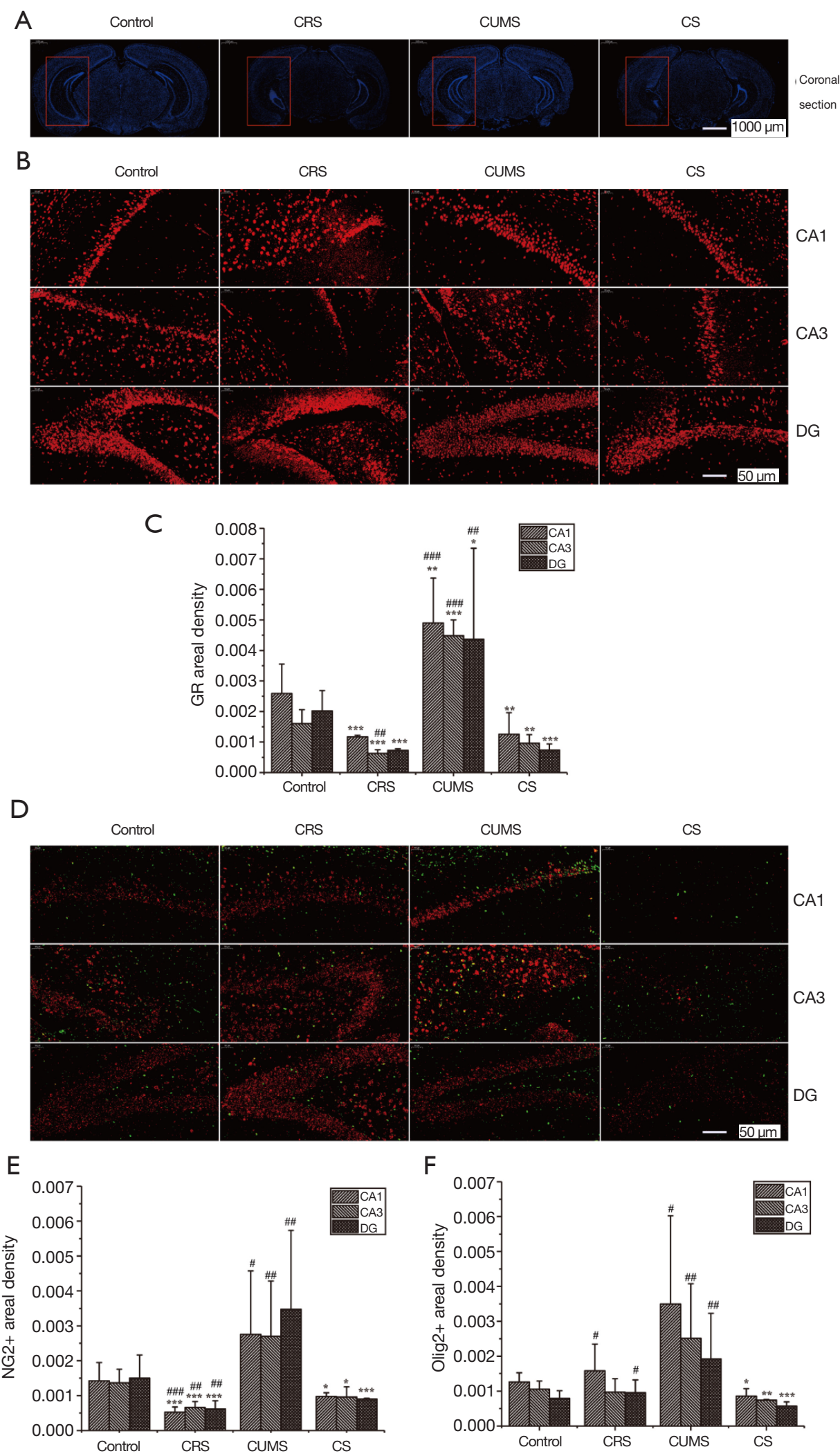


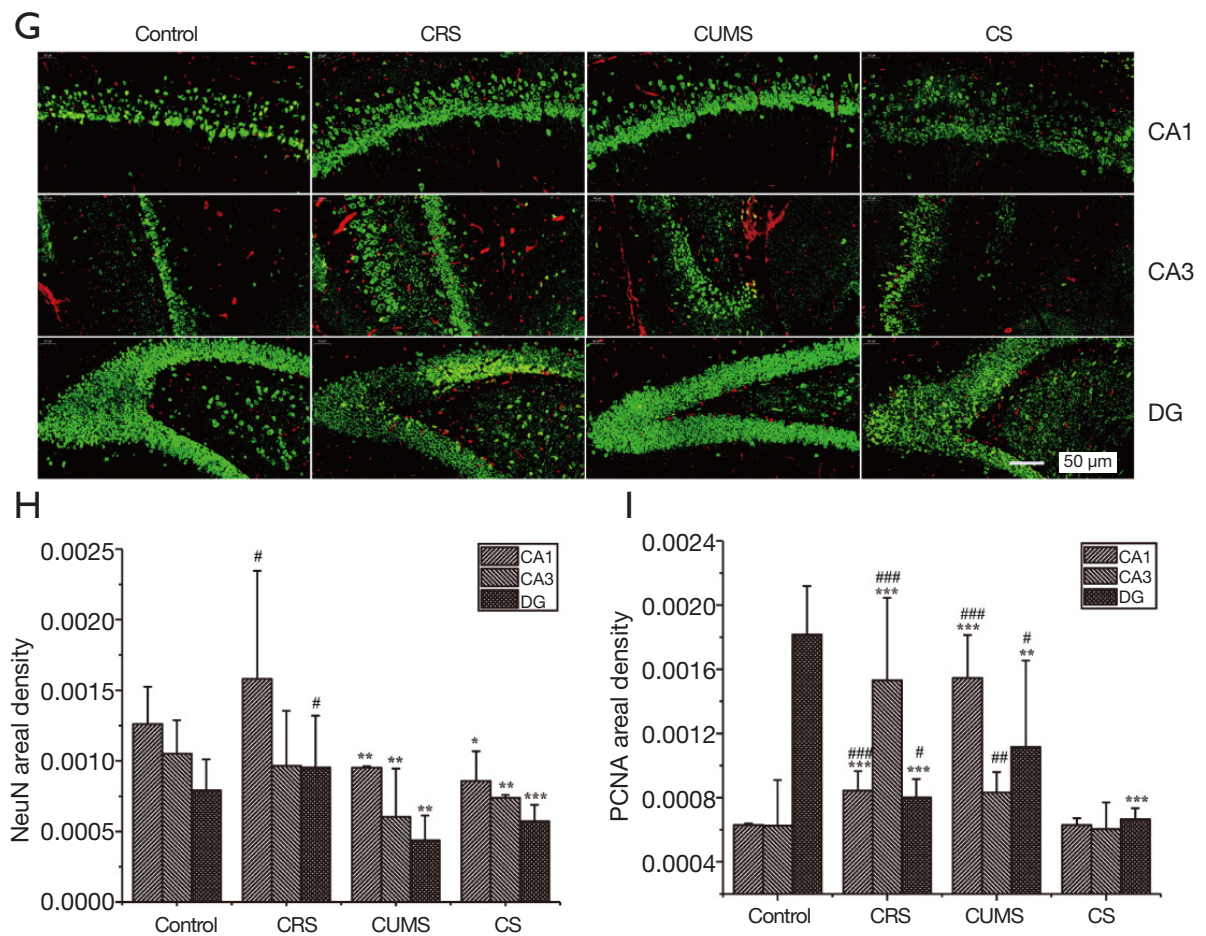

Figure 4 The effect of stress stimulations on structure, expression of GR and oligodendrocytes, and neuron proliferation in key hippocampal subregions. (A) Coronal section image of the mouse brain, (B) immunofluorescence images of GR (red), (C) fluorescence intensity of GR, (D) immunofluorescence images of NG+ (red) and Olig2 + glial cells (green), (E) fluorescence intensity of NG2 + glial cells, (F) fluorescence intensity of Olig2 + glial cells, $(\mathrm{G})$ immunofluorescence images of NeuN+ cells (green) and PCNA+ cells (red), (H) NeuN+ fluorescence intensity, (I) PCNA+ fluorescence intensity in subregions of the hippocampus. Data are shown as mean $\pm \mathrm{SD}(\mathrm{n}=8)$. *, $\mathrm{P}<0.05$, **, $\mathrm{P}<0.01$ and ***, $\mathrm{P}<0.001$ denote significant difference from the control group; ${ }^{\#}, \mathrm{P}<0.05,{ }^{\# \#}, \mathrm{P}<0.01$ and ${ }^{\# \# \#,} \mathrm{P}<0.001$ denote significant difference from the control group.

A

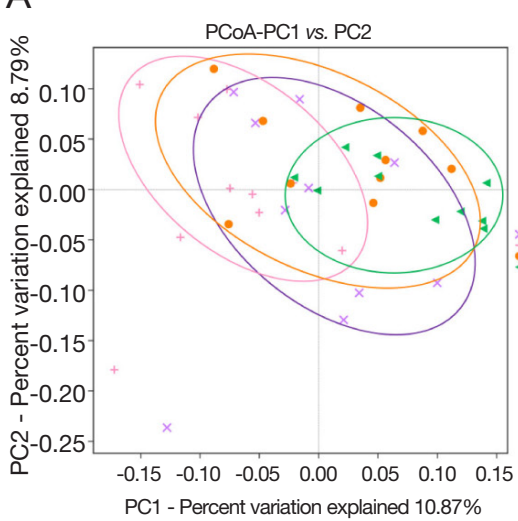

B

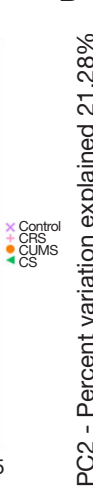

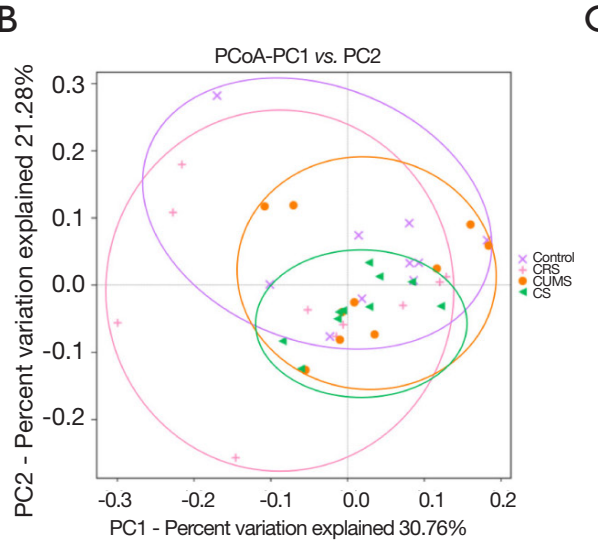

C

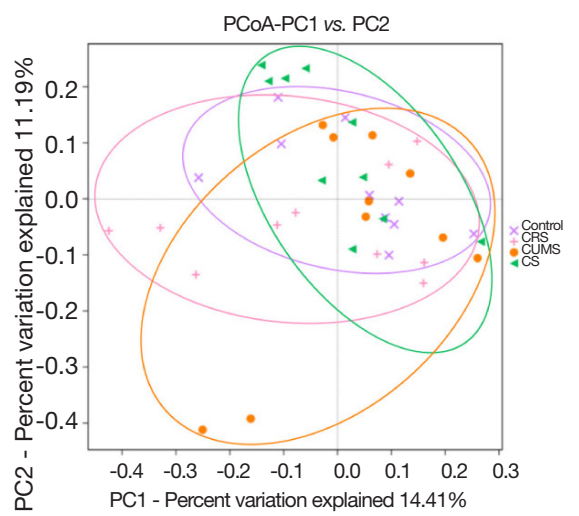

Figure 5 PCoA of gut microbiota beta diversity with the (A) unweighted and (B) weighted UniFrac distances and (C) Bray-Curtis dissimilarity. 
Table 1 Multi-response permutation procedure (MRPP) analysis of gut microbiota.

\begin{tabular}{lccc}
\hline The distance of beta diversity & A value & Observe delta & Expect delta \\
\hline Weighted UniFrac & 0.030 & 0.255 & 0.263 \\
Unweighted UniFrac & 0.045 & 0.338 & 0.018 \\
Bray-Curtis & 0.043 & 0.567 & 0.593 \\
\hline
\end{tabular}
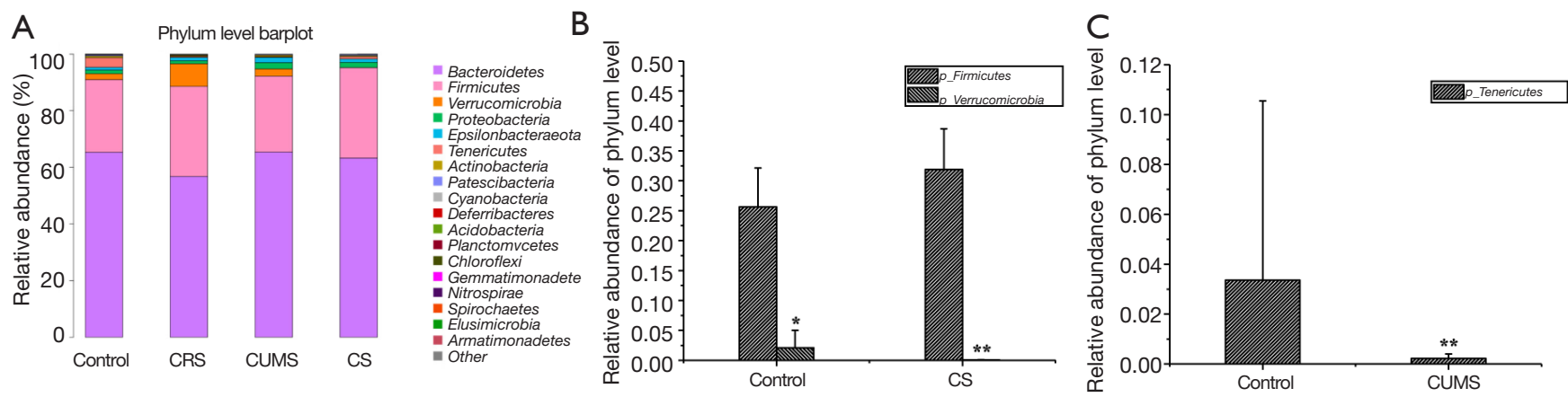

Figure 6 The effect of stress stimulations on the composition of the gut microbiota at the phylum level. (A) The relative abundances of phylum in the intestinal contents of mice after 3 weeks of stress treatment. The comparison of (B) CS group or (C) CUMS group with the control group on the relative abundances of Firmicutes, Verrucomicrobia, and Tenericutes in intestinal contents. Data are shown as mean \pm SD $(\mathrm{n}=10) .{ }^{*}, \mathrm{P}<0.05$ and ${ }^{* *}, \mathrm{P}<0.01$ denote significant difference from the control group.

and CUMS groups, respectively, compared with the control group (Figure 8C,D).

The composition of the gut microbiota at the genus level was investigated, as shown in Figure 9A. Compared with the control group, six genera of gut microbiota were significantly different in relative abundance in the CS group $(\mathrm{P}<0.05$ or $\mathrm{P}<0.01)$, with the relative abundance of Bacteroides, Akkermansia, and Blautia being lower and Lachnospiraceae NK4A136, Rikenella, and Odoribacter being higher than the control group (Figure 9B). Meanwhile, both CRS and CUMS groups had two genera of gut microbiota significantly different from the control group in relative abundance $(\mathrm{P}<0.05$ or $\mathrm{P}<0.01)$, namely, Rikenella and Prevotellaceae UCG-001 in CRS group and Lactobacillus and Mycoplasma in CUMS group (Figure 9C,D).

The changes in the relative abundances of gut microbiota at the baseline are depicted in a cladogram (Figure 10A). According to statistical analysis, f-Polyangiaceae and $\mathrm{f}$-Xanthobacteraceae, f-Bacteroidaceae, and f-Carnobateriaceae were the microbial groups that played an important role in distinguishing the CRS and CUMS groups. At the same time, c-Saccharimonadia, o-Saccharimonadales, f-Saccharimonadaceae, f-Aerococcaceae, and o-Bacillales were the microbial groups that played a key role in distinguishing the CS group. Moreover, according to the histogram of linear discriminant analysis (LDA) scores computed for features differentially abundant among groups (Figure 10B), there were 7, 2, 2, and 4 kinds of gut microbes with LDA value $>2.5$ in the CS, CRS, CUMS, and control groups, respectively.

\section{Discussion}

The stress response can lead to serious illnesses, including depression and post-traumatic stress disorder (20). In the CUMS model, mice are chronically exposed to a bombardment of unpredictable micro-stressors, while CRS deprives mice of freedom as predictable stress (21). Both CRS and CUMS can induce the development of a plethora of behavioral changes, including the decrease of response to rewards and anhedonia (22). However, the effect of predictable stress combined with a bombardment of chronic unpredictable micro-stressors on psychiatric states is not yet fully understood. Hence, we investigated the combined effect of CRS and CUMS on depression-like symptoms in mice. 
A

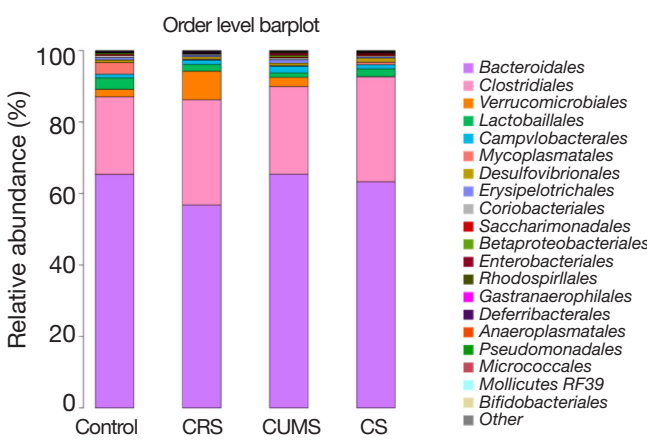

B

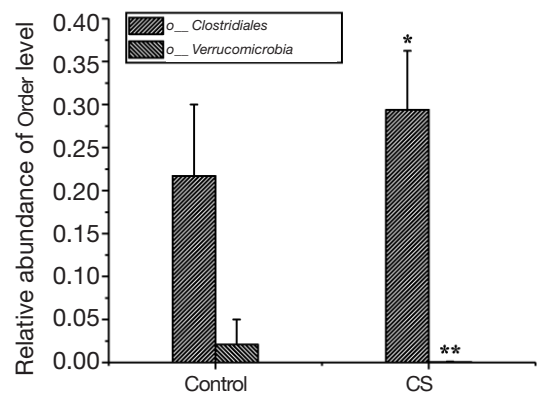

C

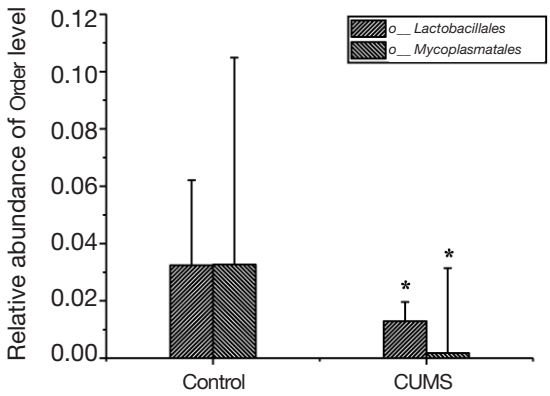

D

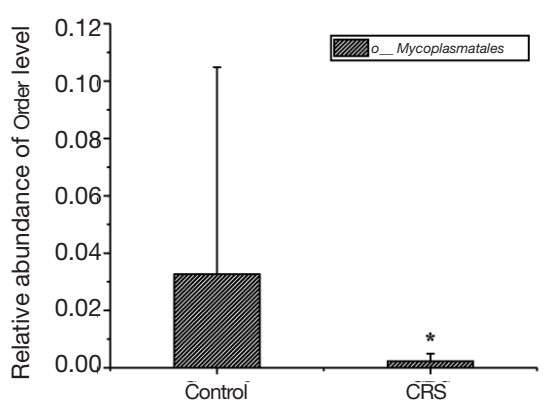

Figure 7 The effect of stress stimulations on the composition of the gut microbiota at the order level. (A) The relative abundances of order in intestinal contents of mice 3 weeks after stress treatment. (B) The comparison of the CS group with the control group on the relative abundances of Clostridiales and Verrucomicrobia in intestinal contents. (C) The comparison of the CRS group with the control group on the relative abundance of Mycoplasmatales in intestinal contents. (D) The comparison of the CUMS group with the control group on the relative abundances of Lactobacillales and Mycoplasmatales in intestinal contents. Data are shown as mean $\pm \mathrm{SD}(\mathrm{n}=10) .{ }^{*}, \mathrm{P}<0.05$ and ${ }^{* *}, \mathrm{P}<0.01$ denote significant difference from the control group. 
A

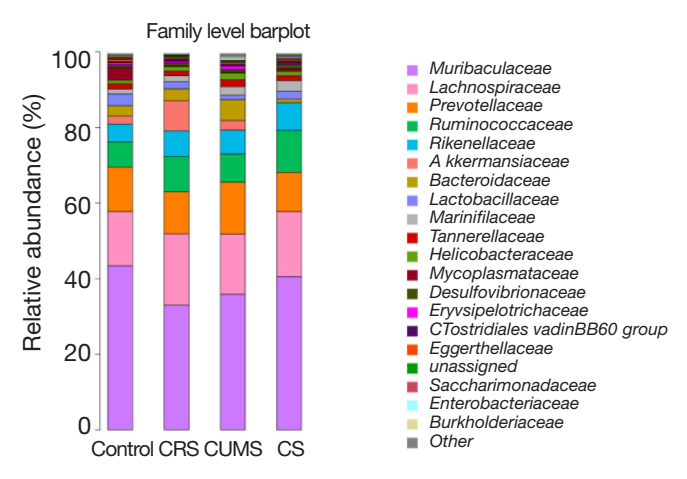

\section{B}
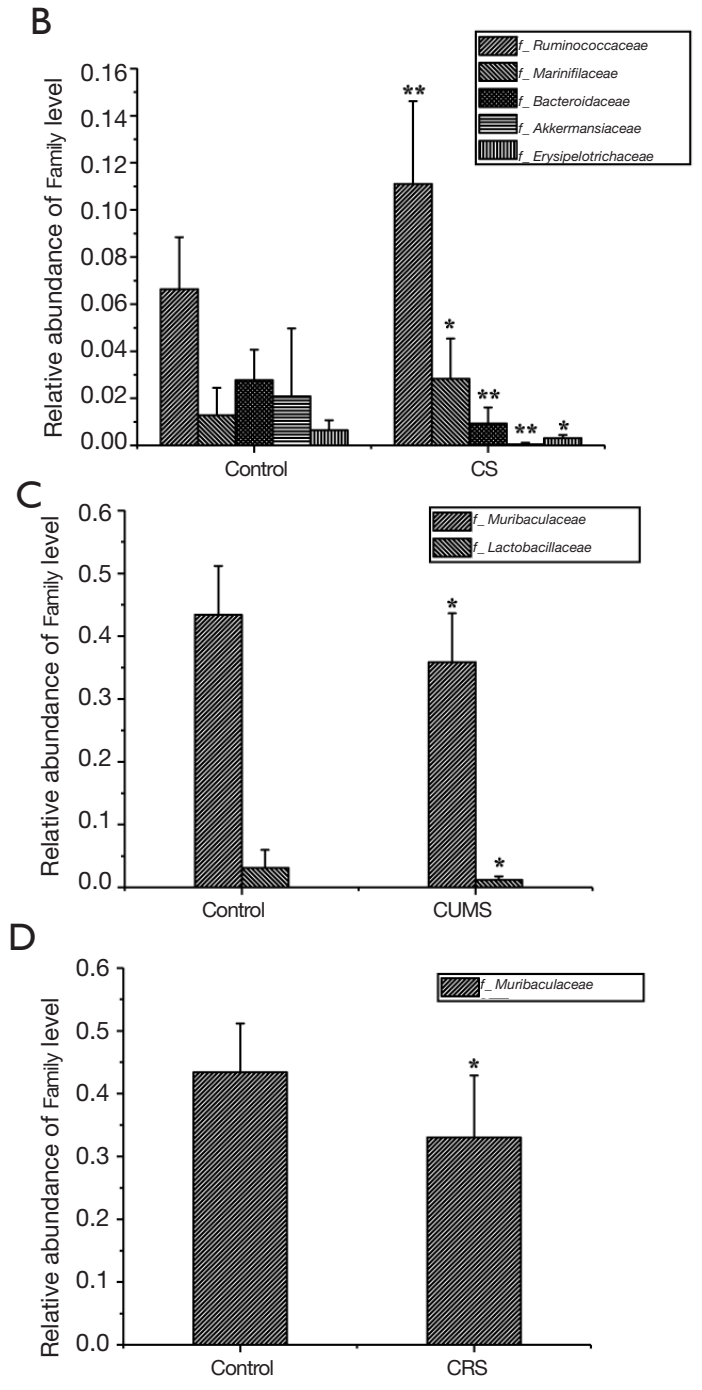

Figure 8 The effect of stress stimulations on the composition of the gut microbiota at the family level. (A) The relative abundances of the family in intestinal contents of mice 3 weeks after stress treatment. (B) The comparison of the CS group with the control group on the relative abundances of Ruminococcaceae, Marinifilaceae, and Bacteroidaceae in intestinal contents. (C) The comparison of the CRS group with the control group on the relative abundance of Muribaculacaea in intestinal contents. (D) The comparison of the CUMS group with the control group on the relative abundances of Muribaculacaea and Lactobacillaceae in intestinal contents. Data are shown as mean $\pm \mathrm{SD}(\mathrm{n}=10)$. *, $\mathrm{P}<0.05$ and ${ }^{* *}, \mathrm{P}<0.01$ denote a significant difference from the control group. 
A

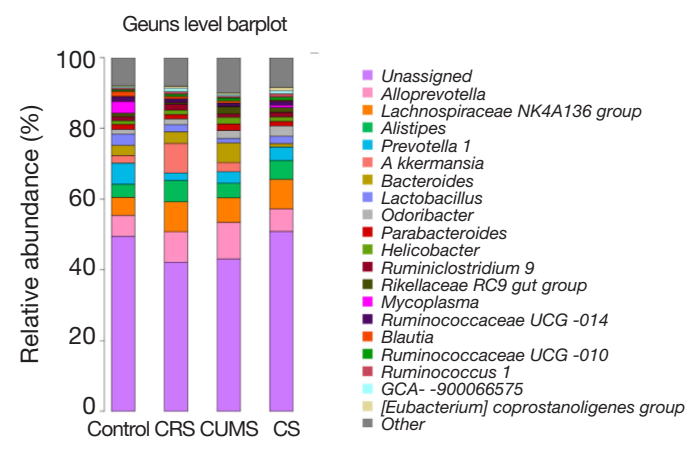

B
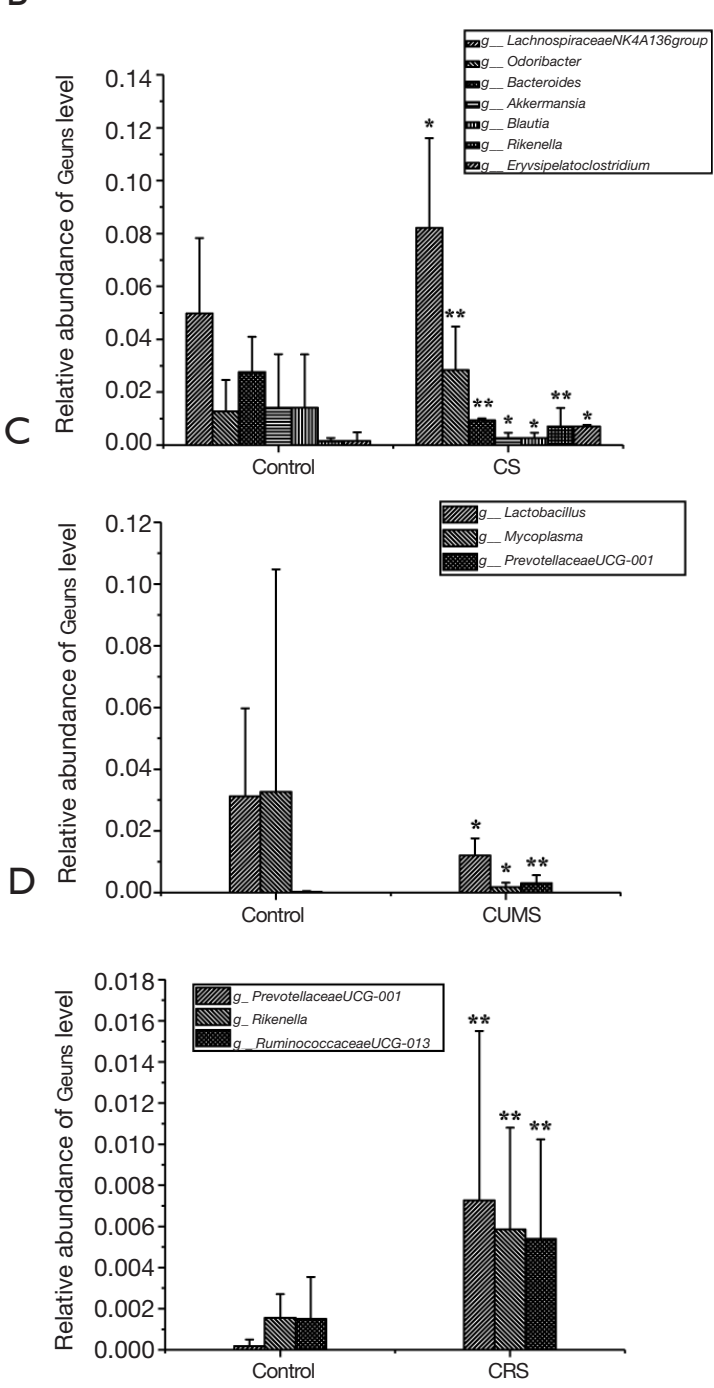

Figure 9 The effect of stress stimulations on the composition of the gut microbiota at the genus level. (A) The relative abundances of the genus in intestinal contents of mice 3 weeks after stress treatment. (B) The comparison of the CS group with the control group on the relative abundances of LachnospiraceaeNK4A136, Rikenella, Odoribacter, Bacteroides, Akkermansia, and Blautia in intestinal contents. (C) The comparison of the CRS group with the control group on the relative abundance of Rikenella and PrevotellaceaeUCG-001 in intestinal contents. (D) The comparison of the CUMS group with the control group on the relative abundances of Lactobacillus and Mycoplasma in intestinal contents. Data are shown as mean $\pm \mathrm{SD}(\mathrm{n}=10)$. *, $\mathrm{P}<0.05$ and $^{* *}, \mathrm{P}<0.01$ denote significant difference from the control group. 
A

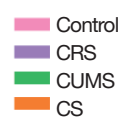

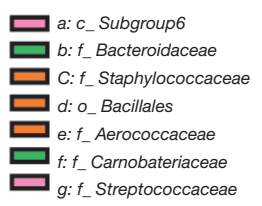

h:f_Saccharimonadaceae

i: o_Saccharimonadales

j:c_Saccharimonadia

k: $f_{-}$Devosiaceae

I: $f_{-}$Xanthobacteraceae

m:f_Polyangiaceae

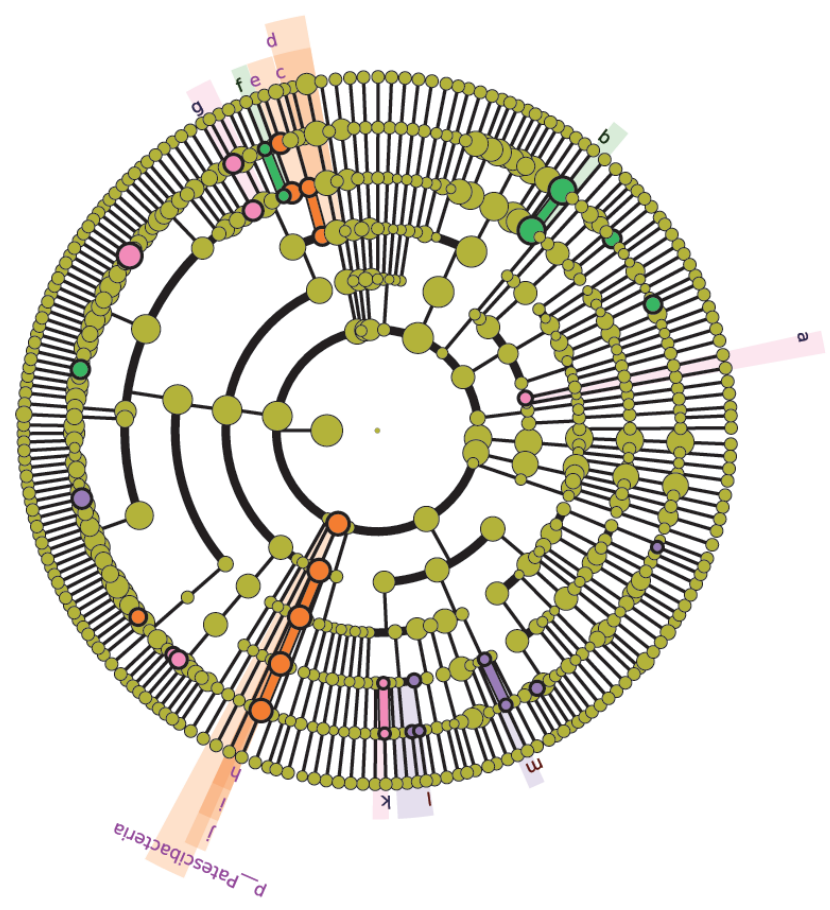

B

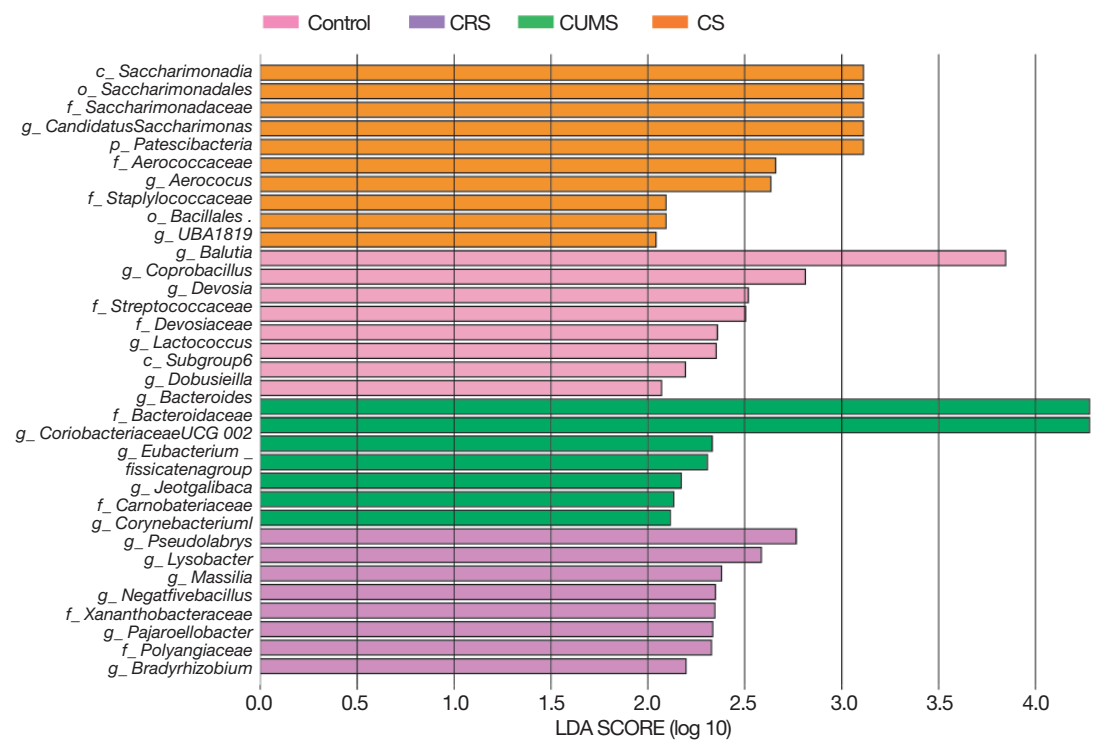

Figure 10 Analysis of species differences between groups. (A) Cladogram representing taxa with different abundances of gut microbiota at the baseline and (B) histogram of linear discriminant analysis scores computed for features differentially abundant among groups. The linear discriminant analysis (LDA) score on the $\log 10$ scale is showed at the bottom. The greater the LDA score is, the more significant the phylotype biomarker is in the comparison. 
Major depressive disorder (MDD) is often associated with appetite changes (loss of appetite or increased appetite) and subsequent weight changes (23). In the present study, after 3 weeks of treatment with combined stress of CRS and CUMS, the mice reduced their food intake and lost weight significantly, with the weight loss being much more significant than that in CRS or CUMS group. As for the core symptom of depressive disorders, anhedonia refers to broadly stunted reactions to rewards or the inability to enjoy happiness, and a decrease in sucrose preference reflects anhedonia of mice in the CS group. Learned helplessness is an essential indicator for assessing depressive-like behavior, and the increase of immobility in FST and TST indicates a lower desire to escape, which may mimic the symptom of helplessness in depression. Moreover, the spontaneous general activity of stressed rats was assayed using OFT, and our result showed that stress significantly reduced the distance moved and center residence time and prolonged the rest time, which indicated that stressed mice are less active.

Despite advances in the understanding of the neurobiology of MDD, currently, no established mechanism can explain all the facets of the disease. However, MDD is associated with alterations in regional brain volumes, particularly the hippocampus (24). Our results showed that the combined stress of CRS and CUMS induced the disordered arrangement of vertebral cells, fewer vertebral cells, and atrophy in the hippocampus of mice, suggesting severe structural damage.

To date, a few theories have been proposed for the pathogenesis of MDD: (I) monoamine hypothesis on the deficiency of the biogenic amine system, particularly 5-HT and NE (25); (II) HPA axis dysfunction theory on the hyperactivity of the HPA system reflected in high levels of neuroendocrine hormones (26); (III) neurogenesis and neurodegenerative hypothesis with the decrease of neurogenesis and occurrence of neurodegeneration $(27,28)$; and (IV) inflammatory theory with peripheral immune dysfunction and neuro-immunological mechanisms (25). Additionally, BDNF and GR have often been suggested to contribute to the pathophysiology of MDD, with BDNF playing a significant role in neuronal growth and survival, serving as a neurotransmitter modulator (29), and contributing to neuronal plasticity. Meanwhile, the reduction of GR could lead to the disorder and hyperactivity of the HPA axis function, finally presenting as abnormal neuroendocrine function and depressive-like behaviors $(26,30)$. Recent studies have found that oxidative stress plays a significant role in the pathophysiology of major depression via actions of free radicals, nonradical molecules, and reactive oxygen and nitrogen species, so products of oxidative stress represent essential parameters for measuring and predicting depression status (31). Our study found that combined CRS and CUMS for 3 weeks caused a reduction in GR level, loss of oligodendrocytes (NG2 and Olig2 cells), and inhibition of neuron proliferation (decrease of PCNA+ and $\mathrm{NeuN}+$ expression) in the CA1, CA3, and DG regions of the hippocampus.

Furthermore, it decreased the levels of monoamine neurotransmitters (5-HT and NE) and BDNF in the cerebral cortex, induced hyperactivity of the HPA system indicated by increased levels of CORT, CRH, and ACTH in serum. Also, it led to immune dysfunction indicated by increased TNF $\alpha$ and decreased IL- 6 and IL-10 levels in serum, aggravated oxidative stress, and weakened the capacity of antioxidants, as indicated by increased MDA and decreased SOD and CAT in serum. Compared with CRS or CUMS alone, combined stress more significantly increased MDA levels, decreased 5-HT, SOD, and CAT levels and inhibited the expressions of Olig2+ and PCNA+ in key subregions of the hippocampus, but had a weaker effect on lowering the $\mathrm{BDNF}$ content in mice.

Gut microbiota is essential to human health, and the immune system and plays a significant role in the bidirectional communication between the gut and the brain (known as the gut-brain axis). More and more evidence has suggested the gut microbiota is associated with neuropsychiatric disorders, including schizophrenia, autistic disorders, anxiety disorders, and major depressive disorder (32). Hence, the diversity of the gut microbiota could aid in understanding depression (33). In the present study, we found that mice exposed to combined stress had fewer probiotics and more harmful bacteria than normal control mice. Moreover, the change in the diversity of the gut microbiota of mice exposed to combined stress was even greater than that of mice exposed to a single type of stress. Compared with the normal control group, the numbers of gut microorganisms with significant changes in relative abundance at phylum, order, family, and genus level were 2, 2, 3, and 6 in the CS group, 0, 1,1, and 2 in the CRS group, and 1,2,2, and 2 in the CUMS group, respectively. Furthermore, there were 7, 2, and 2 kinds of gut microbes with LDA value $>2.5$ in the CS, CRS, and CUMS groups, respectively.

The intestinal tract is an intricate internal environment, and the intestinal flora plays a vital role in maintaining 
the internal environment of the body (34), influencing the metabolic balance of the body, the activation of the immune system (35) and the HPA axis (36), as well as brain activity. Schwarcz et al. (37) found that metabolites of the intestinal microbial community may affect the function of neurotransmitters through the tryptophan metabolism pathway, and these metabolites can also be involved in the inflammatory response and brain activity, but the specific mechanism involved is not precise (38-40). Sudo et al. (41) found that excessive excitement of the HPA axis induced by stress may be associated with some probiotics in mice. Moreover, some studies have found that the probiotic bacteria in the gut microbiome can improve the body's antioxidant capacity, including promoting T-AOC, SOD, and GSHpx levels and reducing MDA levels (42). Moreover, gut microbiota was found to be involved in the development and functionality of microglia and astrocyte, neural development, neurotransmitter immune activation, the central nervous system, and neural, physiological processes, including bloodbrain barrier integrity (40). In the present study, we found that combined stress-induced more severe hippocampal atrophy, oligodendrocytes injury, and oxidative stress in mice than single stress, which was consistent with the change in the diversity of the gut microbiota. Among the gut microorganisms with significant changes in relative abundance, some are associated with depression, including the Bacteroides and Lactobacillus families (32), and autoimmunity, including Saccharimonadaceae, in clinical trials (43). However, the relationship of numerous gut microorganisms with depression is still unknown and requires further study.

In summary, our data suggested that combined stress of predictable CRS and CUMS caused significant weight loss, food intake reduction, depression-like behaviorsincluding anhedonia, learned helplessness, and reduction in spontaneous activity-and even atrophy and severe structural damage to the hippocampus in mice. Our pathogenesis study showed that combined stress-induced the reduction of GR level, loss of oligodendrocytes, and inhibition of neuron proliferation in the CA1, CA3, and DG regions of the hippocampus, decreased the contents of monoamine neurotransmitters and BDNF in the cerebral cortex, caused hyperactivity of HPA, led to immune dysfunction, aggravated oxidative stress, and weakened the capacity of antioxidants in mice. Furthermore, compared with single stress, combined stress gave rise to a more significant change in the diversity of the gut microbiota, which might be one of the reasons for its more significant effect on increasing oxidative stress and decreasing monoamine neurotransmitter content, oligodendrocyte number, and neuron proliferation in mice.

\section{Acknowledgments}

Funding: This work was supported by the National Key R\&D Program of China (2018YFC1708006), China Postdoctoral Science Foundation-funded project (2019M652982), the Innovation Platform Program (2017-ZJ-Y08) and the Natural Science Foundation of Qinghai Province (2018-ZJ-902), the International Partnership Program (153631KYSB20160004) and the Central Asian Drug Discovery and Development Center of CAS (CAM201806), and Youth Innovation Promotion Association CAS.

\section{Footnote}

Reporting Checklist: The authors have completed the ARRIVE reporting checklist. Available at http://dx.doi. org/10.21037/atm-20-5168

Data Sharing Statement: Available at http://dx.doi. org/10.21037/atm-20-5168

Conflicts of Interest: All authors have completed the ICMJE uniform disclosure form (available at http://dx.doi. org/10.21037/atm-20-5168). The authors have no conflicts of interest to declare.

Ethical Statement: The authors are accountable for all aspects of the work in ensuring that questions related to the accuracy or integrity of any part of the work are appropriately investigated and resolved. All of the animal experiments were in compliance with the ARRIVE guidelines, carried out in strict accordance with the National Institutes of Health Guide for the Care and Use of Laboratory Animals (NIH Publication No. 8023, revised 1978), and approved by the committee of the Northwest Plateau Institute of Biology, CAS, for animal experiments (allowance number: NWIPB20171106-01).

Open Access Statement: This is an Open Access article distributed in accordance with the Creative Commons Attribution-NonCommercial-NoDerivs 4.0 International License (CC BY-NC-ND 4.0), which permits the noncommercial replication and distribution of the article with the strict proviso that no changes or edits are made and the 
original work is properly cited (including links to both the formal publication through the relevant DOI and the license). See: https://creativecommons.org/licenses/by-nc-nd/4.0/.

\section{References}

1. Kupfer DJP, Frank EP, Phillips MLM. Major depressive disorder: new clinical, neurobiological, and treatment perspectives. Lancet 2012;379:1045-55.

2. Global Burden of Disease Study 2013 Collaborators. Global, regional, and national incidence, prevalence, and years lived with disability for 301 acute and chronic diseases and injuries in 188 countries, 1990-2013: a systematic analysis for the Global Burden of Disease Study 2013. Lancet 2015;386:743-800.

3. Hamel C, Lang E, Morissette K, et al. Screening for depression in women during pregnancy or the first year postpartum and in the general adult population: a protocol for two systematic reviews to update a guideline of the Canadian Task Force on Preventive Health Care. Systematic Reviews 2019;8:27.

4. Targum SD, Fava M. Fatigue as a Residual Symptom of Depression. Innov Clin Neurosci 2011;8:40-3.

5. Bressington D, Mui J, Yu C, et al. Feasibility of a groupbased laughter yoga intervention as an adjunctive treatment for residual symptoms of depression, anxiety and stress in people with depression. J Affect Disord 2019;248:42-51.

6. Marsay C, Manderson L, Subramaney U. Changes in mood after screening for antenatal anxiety and depression. J Reprod Infant Psychol 2018;36:347-62.

7. Touma C, Bunck M, Glasl L, et al. Mice selected for high versus low stress reactivity: A new animal model for affective disorders. Psychoneuroendocrinology 2008;33:839-62.

8. Rose J, Rillich J, Stevenson PA. Chronic social defeat induces long-term behavioral depression of aggressive motivation in an invertebrate model system. PLoS One 2017;12:1-16.

9. Hu C, Luo Y, Wang H, et al. Re-evaluation of the interrelationships among the behavioral tests in rats exposed to chronic unpredictable mild stress. PLoS One 2017;12:e0185129.

10. Han A, Sung Y, Chung S, et al. Possible additional antidepressant-like mechanism of sodium butyrate: Targeting the hippocampus. Neuropharmacology 2014;81:292-302.

11. Eiland L, Ramroop J, Hill MN, et al. Chronic juvenile stress produces corticolimbic dendritic architectural remodeling and modulates emotional behavior in male and female rats. Psychoneuroendocrinology 2012;37:39-47.

12. Ma L, Xu Y, Wang G, et al. What do we know about sex differences in depression: A review of animal models and potential mechanisms. Prog Neuropsychopharmacol Biol Psychiatry 2019;89:48-56.

13. Chiba S, Numakawa T, Ninomiya M, et al. Chronic restraint stress causes anxiety- and depression-like behaviors, downregulates glucocorticoid receptor expression, and attenuates glutamate release induced by brain-derived neurotrophic factor in the prefrontal cortex. Prog Neuropsychopharmacol Biol Psychiatry 2012;39:112-9.

14. Antoniuk S, Bijata M, Ponimaskin E, et al. Chronic unpredictable mild stress for modeling depression in rodents: Meta-analysis of model reliability. Neurosci Biobehav Rev 2019;99:101-16.

15. Dang H, Chen Y, Liu X, et al. Antidepressant effects of ginseng total saponins in the forced swimming test and chronic mild stress models of depression. Prog Neuropsychopharmacol Biol Psychiatry 2009;33:1417-24.

16. Choleris E, Thomas AW, Kavaliers M, et al. A detailed ethological analysis of the mouse open field test: effects of diazepam, chlordiazepoxide and an extremely low frequency pulsed magnetic field. Neurosci Biobehav Rev 2001;25:235-60.

17. Steru L, Chermat R, Thierry B, et al. The tail suspension test: a new method for screening antidepressants in mice. Psychopharmacology (Berl) 1985;85:367-70.

18. Porsolt RD, Bertin A, Jalfre M. Behavioral despair in mice: a primary screening test for antidepressants. Arch Int Pharmacodyn Ther 1977;229:327-36.

19. Amato KR, Yeoman CJ, Kent A, et al. Habitat degradation impacts black howler monkey (Alouatta pigra) gastrointestinal microbiomes. ISME J 2013;7:1344-53.

20. Anisman H. Cascading effects of stressors and inflammatory immune system activation: implications for major depressive disorder. J Psychiatry Neurosci 2009;34:4-20.

21. Wang Q, Timberlake MA 2nd, Prall K, et al. The recent progress in animal models of depression. Prog Neuropsychopharmacol Biol Psychiatry 2017;77:99-109.

22. Harro J. Animal models of depression: pros and cons. Cell Tissue Res 2019;377:5-20.

23. Fava M. Weight gain and antidepressants. J Clin Psychiatry 2000;61 Suppl 11:37-41.

24. Almansa R, Nogales L, Martín-Fernández M, et al. 
Transcriptomic depression of immunological synapse as a signature of ventilator-associated pneumonia. Ann Transl Med 2018;6:415.

25. Ng J, Papandreou A, Heales SJ, et al. Monoamine neurotransmitter disorders--clinical advances and future perspectives. Nat Rev Neurol 2015;11:567-84.

26. Pariante CM, Lightman SL. The HPA axis in major depression: classical theories and new developments. Trends Neurosci 2008;31:464-8.

27. Sapolsky RM. Is impaired neurogenesis relevant to the affective symptoms of depression? Biol Psychiat 2004;56:137-9.

28. Czéh B, Lucassen PJ. What causes the hippocampal volume decrease in depression? Are neurogenesis, glial changes and apoptosis implicated?. Eur Arch Psychiatry Clin Neurosci 2007;257:250-60.

29. Kishi T, Yoshimura R, Ikuta T, et al. Brain-Derived Neurotrophic Factor and Major Depressive Disorder: Evidence from Meta-Analyses. Front Psychiatry 2018;8:308.

30. Zunszain PA, Anacker C, Cattaneo A, et al. Glucocorticoids, cytokines and brain abnormalities in depression. Prog Neuropsychopharmacol Biol Psychiatry 2011;35:722-9.

31. Vaváková M, Ďuračková Z, Trebatická J. Markers of Oxidative Stress and Neuroprogression in Depression Disorder. Oxid Med Cell Longev 2015;2015:898393.

32. Evrensel A, Ceylan ME. The Gut-Brain Axis: The Missing Link in Depression. Clin Psychopharmacol Neurosci 2015;13:239-44.

33. Pusceddu MM, El Aidy S, Crispie F, et al. N-3 Polyunsaturated Fatty Acids (PUFAs) Reverse the Impact of Early-Life Stress on the Gut Microbiota. PLoS One 2015;10:e139721.

Cite this article as: Qiao Y, Zhao J, Li C, Zhang M, Wei L, Zhang X, Kurskaya O, Bi H, Gao T. Effect of combined chronic predictable and unpredictable stress on depressionlike symptoms in mice. Ann Transl Med 2020;8(15):942. doi: 10.21037/atm-20-5168
34. Agus A, Planchais J, Sokol H. Gut Microbiota Regulation of Tryptophan Metabolism in Health and Disease. Cell Host Microbe 2018;23:716-24.

35. Maslowski KM, Mackay CR. Diet, gut microbiota and immune responses. Nat Immunol 2011;12:5-9.

36. Sudo, N. Microbiome, HPA axis and production of endocrine hormones in the gut. Adv Exp Med Biol 2014;817:177-94.

37. Schwarcz R, Bruno JP, Muchowski PJ, et al. Kynurenines in the mammalian brain: when physiology meets pathology. Nat Rev Neurosci 2012;13:465-77.

38. O'Mahony SM, Marchesi JR, Scully P, et al. Early Life Stress Alters Behavior, Immunity, and Microbiota in Rats: Implications for Irritable Bowel Syndrome and Psychiatric Illnesses. Biol Psychiat 2009;65:263-7.

39. Mayer EA. Gut feelings: the emerging biology of gutbrain communication. Nat Rev Neurosci 2011;12:453-66.

40. Maqsood R, Stone TW. The Gut-Brain Axis, BDNF, NMDA and CNS Disorders. Neurochem Res 2016;41:2819-35.

41. Sudo N, Chida Y, Aiba Y, et al. Postnatal microbial colonization programs the hypothalamic-pituitaryadrenal system for stress response in mice. J Physiol 2004;558:263-75.

42. Li A, Wang Y, Li Z, et al. Probiotics isolated from yaks improves the growth performance, antioxidant activity, and cytokines related to immunity and inflammation in mice. Microb Cell Fact 2019;18:112.

43. Russell JT, Roesch LFW, Ördberg M, et al. Genetic risk for autoimmunity is associated with distinct changes in the human gut microbiome. Nat Commun 2019;10:3621.

(English Language Editor: J. Chapnick) 